\title{
Evaluation of programmed death 1 (PD-1) and programmed death ligand- 1 (PDL1) as tumor biomarkers an in patients with head and neck squamous cell carcinoma
}

\author{
Elitsa Deliverska ${ }^{1}$, Payam Forghani ${ }^{2}$, Sadeta Parusheva ${ }^{1}$ \\ 1. Department of Dental, Oral and Maxillofacial surgery, \\ Faculty of Dental Medicine, Medical University of Sofia;
}

2. Student in Faculty of Dental Medicine, Medical University of Sofia;

\section{Abstract}

The use of onco-biomarkers such as programmed death 1 (PD-1) and its ligand, PD-L1, present a new opportunity for the diagnosis and treatment of head and neck squamous cell carcinomas (HNSCC). These molecules modulate the immune response of the host and aid the tumor cells to escape immune surveillance. The overexpression of PD-1/PD-L1 in some HNSCC is a predictive biomarker to the response of checkpoint inhibitor immunotherapeutic drugs.

Keywords: PDL-1, maxillofacial, squamous cell carcinoma, biomarkers, checkpoint inhibitors 


\section{Background}

Oral squamous cell carcinoma is one of the most common malignancies affecting the head and neck region. The incidence of head and neck squamous cell carcinoma (HNSCC) has gradually increased over the past decades, they are the 6th most common malignancy with 600,000 new cases annually. (1) HNSCC encompasses a heterogenous group of tumors that arise from the squamous epithelium of the oral cavity, oropharyngs, larynx and hypopharynx. HNSCC are sometimes referred to as treatment resistant tumors, with unfavorable patient outcomes and survival rates. (2) Smoking and alcohol are regarded as the classical etiological factors of HNSCC, however, a clinically, genomically and immunologically separate subcategory of tumors arise from the epithelium of the tonsils and the base of tongue as a result of infection with Human Papilloma Virus (HPV). The prognosis and treatment modalities differ pointedly in HPV(+)and HPV(-) HNSCC tumors. (3)

Surgery is the conventional treatment for HNSCC despite the swift developments in treatment variants such as chemotherapy, radiotherapy and target therapy, while some patients with advanced cases are administered palliative-intent therapy. Immune evasion remains one of the leading challenges affecting the successful treatment of cancer. Numerous biomarkers have been linked with the diagnosis and prognosis of HNSCC. Immunohistochemical staining of tumor cells reveal PD-1 and its ligand, programmed deathligand 1 (PD-L1), as a diagnostic biomarker and a potential treatment method for HNSCC. $(4,5)$ PD-L1 is an immune co-stimulatory molecule that belongs to the $\mathrm{B} 7-\mathrm{H}$ gene family. It is expressed on countless tumor cell types and the surface of immune cells, comprising B cells, $T$ cells, myeloid dendritic cells and macrophages. (6) PD-L1 is expressed in approximately 79\% of patients with HNSCC. PD-L1 plays an imperative role in modifying cellular immunity, its expression stimulates glycolytic metabolism through metabolic competition between tumor cells and tumor-infiltrating lymphocytes within the tumor microenvironment. PD-L1 is regulated by a close interrelation between tumor and stromal cells within the tumor micro-environment. PD-L1 is a critical inhibitor of immune activation and plays a critical role in tumor evasion of anti-tumor immune responses by modulating the host immune system. $(7,8)$ PD-1 gene polymorphisms are associated with autoimmune diseases, including lupus erythematosus and rheumatoid arthritis. It is responsible for the initiation and preservation of immune suppression within the tumor microenvironment (TME). The expression of PD-L1 gradually escalates together along with the increase in tumor grades. (9)

PD-L1 is located in the cell membrane and cytoplasm of tumor cells and infiltrating lymphocytes, as well as physiologically in the placenta, pancreatic islet cells and mesenchymal stem cells. (10) High PD-L1 expression is associated with poor differentiation of tumor, nodal metastasis, advanced stage, and distant metastasis. PD-L1 expression is affected by the surrounding microenvironment. Some inflammatory conditions, hypoxia or metabolic activity within the tumor microenvironment have been shown to change PD-L1 expression in tumor cells. PD-1 expression is upregulated in response to pro-inflammatory cytokines IFN- $\gamma$, TNF- $\alpha$, and IL-1 $\beta$. IFN- $\gamma$ produced by T-cells and IL-1 $\beta$ upregulates, induces and enhances PD-L1 expression in tumor cells. (11-13) There exists an inverse association between PD-L1 expression and vascular invasion with the TME. Characteristics of vascular invasion include intratumoral angiogenesis and release of vascular endothelial growth factor. Regarding the prognostic value of PD-L1 in tumor cells, PDL1 expression in HNSCC as a prognostic significance is controversial. In some studies, it has been associated with poor prognosis, better prognosis or not associated with prognosis. (14) PD-1 levels are higher in tongue SCC than in lip SCC, with the latter having a better prognosis. This can be explained by the local lymphatic and blood supply of tongue, generating better nutrition and possibility of tumor invasiveness. Several studies show that PD-L1 overexpressing (PD-L1+) tumors may have a poorer 
prognosis than PD-L1 negative (PD-L1-) tumors, though, PD-L1+ tumors are also more sensitive to antiPD-L1 immunotherapeutic agents. (15-18)

Tumors can escape the host immune surveillance through numerous mechanisms, that is why combination therapy is the golden treatment option. PD1 and PD-L1 inhibit T-cell activation and downgrade antitumor response. PD-1 on antigen-specific T cells binds with PD-L1 on tumor cells, resulting in the exhaustion of antigen specific $T$ cells and prompts apoptosis of antigen-specific T cells. PD-L1 binding to tumor cells and PD-1 on regulatory T cells, stimulates immune tolerance of immune system to cancer cells. (18-20) Modern targeted immunotherapeutic drugs on the market aim to reactivate immune surveillance and elicit antitumor activity.

Checkpoint inhibitors as PD-L1 on tumour cells and PD-1 on T cells can help to keep the accurate immune system response. Binding of PD-L1 to PD-1 didn't allow T cells to kill the tumour cells and blocking that binding by immune checkpoints inhibitors (anti PD-1 and anti PDL-1) allows tumour cells to be killed by T cells.

Anti- PD-L1 monoclonal antibodies (A PD-L1) are effective in oncotherapy, and anti-tumor immunity may be enhanced by inhibiting the expression of PD-L1. Pembrolizumab is a humanized monoclonal antibody that blocks the interaction of PD-1 with its ligand, PD-L1. PD-1/PD-L1 checkpoint blockade can extend the survival time of patients with recurrent-metastatic HNSCC by permitting the reinvigorated lymphocyte proliferation and effector function. Anti-PD1 antibody Nivelumab has been proven to increase overall survival of patients. These checkpoint blockades show outstanding clinical value, durable response and minimal toxicity. However, the effectiveness of PD-1-targeted-therapy is very low as a monotherapy, therefore, it must be used together in combination with other conventional therapies. (21) There exists synergistic activity between the checkpoint inhibitors and radiation. It is imperative to identify the most beneficial timing for combined radiotherapy and immunotherapy, albeit, the concurrent administration equals higher toxicity. Gene analysis can identify patients that will benefit from PD-1 and PD-L1 checkpoint blockades. It is important to find predictive biomarkers for the response to these drugs in order to avoid autoimmune adverse effects and mitigate the high cost of such immunotherapeutic agents. Studies show that elevated levels of PD-1 can be detected also in peripheral blood. Over-expression of PD-L1 is significantly associated with better response to PD-1 and PD-L1 blockade. $(22,23)$ PD-L1 expression was significantly higher among HPV+ nonsmokers. The HPV status is of prognostic importance; nonetheless, studies show the possibility of better prognosis in nonsmokers. (24) HPV + HNSCC is associated with improved prognosis as compared to HPV negative HNSCC. (25) Therefore, HPV + nonsmokers are a good candidate group for treatment of PD1 immunotherapeutic agents. Patients with PD-L1 negative tumors can still derive benefit from therapy pursuing this axis. Immunotherapeutic agents targeting PD-1/PD-L1 molecule via checkpoint inhibition pathway can plausibly dramatically enhance tumor susceptibility to radiation therapy.

\section{Conclusion}

The heteregenous nature of HNSCC tumors add a level of complexity to isolate and strategize a simple treatment protocol and a single drug to the treatment of HNSCC patients. Further research is required in order to identify patients whom can benefit from the combined therapy and to recognize the prognostic significance of PD-L1 expression in the tumor microenvironment. PD-1 represents a promising biomarker that can significantly help in treatment choice amongst therapeutic alternatives. 


\section{References}

1. Addeo R, Caraglia M, Iuliano G. Pembrolizumab: the value of PDL1 biomarker in head and neck cancer, Expert Opinion on Biological Therapy, 2016,16:9, 1075-1078, DOI: 10.1080/14712598.2016.1211635

2. de Souza Malaspina T, Gasparoto, TH, Costa, MRSN, de Melo EF, Ikoma MRV, Damante JH, Cavassani KA, Garlet GP, da Silva JS, Campanelly AP. Enhanced programmed death 1 (PD-1) and PD-1 ligand (PD-L1) expression in patients with actinic cheilitis and oral squamous cell carcinoma. Cancer Immunol Immunother 60, 965 (2011). https://doi.org/10.1007/s00262-011-1007-5

3. Meng X, Huang Z, Teng F, Xing L, Yu J. Predictive biomarkers in PD-1/PD-L1 checkpoint blockade immunotherapy; Anti-tumor treatment; volume 42, issue 10,2015, P868-876.

4. Straub M, Drecoll E, Pfarr N, Weichert W, Langer R, Hapfelmeier A, Götz C, Wolff K, Kolk A, Specht K. CD274/PD-L1 gene amplification and PD-L1 protein expression are common events in squamous cell carcinoma of the oral cavity. Oncotarget. 2016; 7: 12024-12034.

5. Kolk A, Jubitz N, Mengele K, Mantwill K, Bissinger O, Schmitt M, Kremer M and Holm PS. Expression of $Y$-box- binding protein YB-1 allows strati cation into long- and short-term survivors of head and neck cancer patients. British Journal of Cancer. 2011; 105:1864-1873.

6. Badoual C, Hans S, Merillon N, Van Ryswick C, Ravel P, Benhamouda N, Levionnois E, Nizard M, SiMohamed A, Besnier N, Gey A, Rotem-Yehudar R, Pere H, Tran T, Guerin CL, Chauvat A, et al. PD-1expressing tumor- in Itrating T cells are a favorable prognostic biomarker in HPV-associated head and neck cancer. Cancer Res. 2013; 73:128-138.

7. Denaro N, Russi EG, Adamo V, Merlano MC. State-of-the- art and emerging treatment options in the management of head and neck cancer: news from 2013. Oncology. 2014; 86: 212-29. doi: $10.1159 / 000357712$.

8. Pardoll DM. The blockade of immune checkpoints in cancer immunotherapy. Nat Rev Cancer. 2012; 12: 252-64. doi: $10.1038 / n r c 3239$.

9. Müller T, Braun M, Dietrich D, Aktekin S, Höft S, Kristiansen G, Göke F, Schröck A, Brägelmann J, Held S. AE, Bootz F, Brossart P. PD-L1: a novel prognostic biomarker in head and neck squamous cell carcinoma. Oncotarget. 2017; 8: 52889-52900. Retrieved from https://www.oncotarget.com/article/17547/

10. Merlano MC, Monteverde M, Colantonio I, Denaro N, Lo Nigro C, Natoli G, Giurlanda F, Numico G, Russi E. Impact of age on acute toxicity induced by bio- or chemo-radiotherapy in patients with head and neck cancer. Oral Oncol. 2012; 48: 1051-7. doi: 10.1016/j. oraloncology.2012.05.001.

11. Solomon B, Seminars in Cancer Biology (2018),https://doi.org/10.1016/j.semcancer.2018.01.008

12. Zandberg DP, Strome SE. The role of the PD-L: PD-1 pathway in squamous cell carcinoma of the head and neck. Oral Oncol (2014), http://dx.doi.org/10.1016/j.oraloncology.2014.04.003

13. Ritprajak P, Azuma M. Intrinsic and extrinsic control of expression of the immunoregulatory molecule PD-L1 in epithelial cells and squamous cell carcinoma. Oral Oncol 51: 221-228, 2015.

14. Huang W, Zhou X, Liao Q, et al. Clinicopathological and prognostic significance of PD-1/PD-L1 axis expression in patients with tongue squamous cell carcinoma. J Cell Physiol. 2020;1-12. https://doi.org/10.1002/jcp.29590

15. Lilja-Fischer J K, Eriksen J G, Georgsen J B, Vo T T, Larsen S R., Cheng J, Busch-Sørensen M, AuroraGarg D, Steiniche T, Overgaard J. Prognostic impact of PD-L1 in oropharyngeal cancer after primary curative radiotherapy and relation to HPV and tobacco smoking, Acta Oncologica, 2020, DOI: 10.1080/0284186X.2020.1729407

16. Yu D, Liu X, Han G, Liu Y, Zhao X, Wang D, Bian X, Gu T. The let-7 family of microRNAs suppresses immune evasion in head and neck squamous cell carcinoma by promoting PD-L1 degradation. Cell Commun Signal 17, 173 (2019). https://doi.org/10.1186/s12964-019-0490-8

17. Ngamphaiboon N, Chureemas T, Siripoon T, Arsa L, Trachu N, Jiarpinitnun C, Pattaranutaporn P, Sirachainan E, Larbcharoensub $\mathrm{N}$. Characteristics and impact of programmed death-ligand 1 expression, CD8+ tumor-infiltrating lymphocytes, and p16 status in head and neck squamous cell carcinoma. Med Oncol. 2019;36(2):21. 
18. Nagasaka M, Zaki M, Kim H, Raza SN, Yoo G, Lin HS, Sukari A. PD1/PD-L1 inhibition as a potential radiosensitizer in head and neck squamous cell carcinoma: a case report. J Immunother Cancer. 2016 Nov 15;4:83. doi: 10.1186/s40425-016-0187-0. PMID: 27895920; PMCID: PMC5109767.

19. Shi Y. Regulatory mechanisms of PD-L1 expression in cancer cellsCancer Immunol Immunother 67(10): 1481-1489, 2018. PMID: 30120503. DOI: 10.1007/s00262-018-2226-9

20. Matsubara T, Haratake N, Akamine T, Takamori S, Katsura M, Shoji F, Honda H, Oda Y and Maehara Y: Metabolic characteristics of programmed cell death-ligand 1-expressing lung cancer on 18Ffluorodeoxyglucose positron emission tomography/computed tomography. Cancer Med 6(11): 25522561, 2017. PMID: 28980429. DOI: 10.1002/cam4.1215

21. Tang $\mathrm{H}$, Zhou $\mathrm{X}, \mathrm{Ye} \mathrm{Y}, \mathrm{Zhou} \mathrm{Y}, \mathrm{Wu} \mathrm{C}, \mathrm{Xu} \mathrm{Y}$, The different role of PD-L1 in head and neck squamous cell carcinomas: A meta-analysis Pathology - Research and Practice, January 2020, vol. 216,issue 1, 152768, https://doi.org/10.1016/j.prp.2019.152768

22. Tojyo I, Shintani Y, Nakanishi T, Okamoto K, Hiraishi Y, Fujita S, Enaka M, Sato F, Muragaki Y. PD-L1 expression correlated with p53 expression in oral squamous cell carcinoma. Maxillofac Plast Reconstr Surg 41, 56 (2019). https://doi.org/10.1186/s40902-019-0239-8

23. Lin YM, Sung WW, Hsieh MJ, Tsai SC, Lai HW, Yang SM et al (2015) High PD- L1 Expression Correlates with Metastasis and Poor Prognosis in Oral Squamous Cell Carcinoma. PLoS ONE 10(11): e0142656. https://doi.org/10. 1371/journal.pone.0142656

24. Montler R, Bell RB, Thalhofer C, Leidner R, Feng Z, Fox BA, Cheng AC, Bui TG, Tucker C, Hoen H, Weinberg A. OX40, PD-1 and CTLA-4 are selectively expressed on tumor-infiltrating $T$ cells in head and neck cancer. Clin Transl Immunol. 2016;5(4):e70.

25. Malaspina, TS, Gasparoto, TH, Costa, MR, de Melo, EF, Ikoma, MR,Damante, JH, Campanelli, AP. Enhanced programmed death 1 (PD-1) and PD-1 ligand (PD-L1) expression in patients with actinic cheilitis and oral squamous cell carcinoma. Cancer Immunology, Immunotherapy, 2011, 60(7), 965974.

\section{Corresponding author:}

Elitsa Deliverska,

Faculty of Dental Medicine, Medical University of Sofia,

Department of Dental, Oral and Maxillofacial Surgery,

Sofia 1431 Str. "Georgi Sofiiski 1" 